\title{
Enantioreversal in the Sharpless Asymmetric Epoxidation Reaction Controlled by the Molecular Weight of a Covalently Appended Achiral Polymer
}

\section{Supporting Information}

\author{
Neal N. Reed, Tobin J. Dickerson, Grant E. Boldt, and Kim D. Janda* \\ Department of Chemistry and The Skaggs Institute for Chemical Biology \\ The Scripps Research Institute \\ 10550 North Torrey Pines Road \\ La Jolla, CA 92037 USA \\ Telephone: +18587842516 \\ Fax: +1 8587842595 \\ Email: kdjanda@scripps.edu
}

Table of Contents:

General Methods

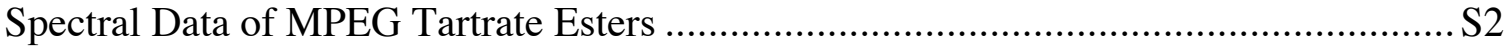

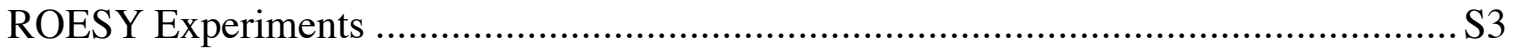

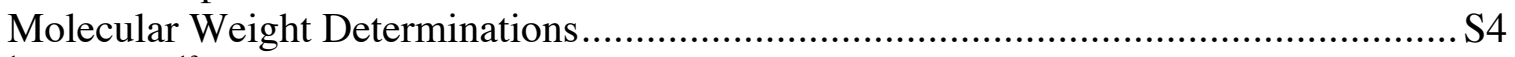

${ }^{1} \mathrm{H}$ NMR, ${ }^{13} \mathrm{C}$ NMR, and MS Data of all MPEG Tartrate Esters .................................. S5 
General Methods. Unless otherwise stated, all reactions were performed under an inert atmosphere with dry reagents and solvents and flame-dried glassware. Analytical thin-layer chromatography (TLC) was performed using $0.25 \mathrm{~mm}$ pre-coated silica gel Kieselgel $60 \mathrm{~F}_{254}$ plates. Visualization of the chromatogram was by UV absorbance, iodine, dinitrophenylhydrazine, ceric ammonium molybdate, ninhydrin or potassium permanganate as appropriate. Preparative and semi-preparative TLC was performed using Merck $1 \mathrm{~mm}$ or $0.5 \mathrm{~mm}$ coated silica gel Kieselgel $60 \mathrm{~F}_{254}$ plates respectively. Analytical HPLC was performed on a Hitachi L-5000 series HPLC using a Vydac $201 T P 54$ reversed phase $\mathrm{C}_{18}$ column. Methylene chloride and chloroform were distilled from calcium hydride. Tetrahydrofuran (THF) was distilled from sodium/benzophenone. Methanol was distilled from magenesium. ${ }^{1} \mathrm{H}$ spectra were recorded on either a Bruker DRX-500 or DRX-600 spectrometer at $500 \mathrm{MHz}$ or $600 \mathrm{MHz}$ respectively and are reported in ppm, unless otherwise noted. ${ }^{13} \mathrm{C}$ NMR were recorded on either a Bruker DRX-500 or DRX-600 spectrometer at $125 \mathrm{MHz}$ or $150 \mathrm{MHz}$ respectively and are reported in ppm, Matrix-assisted laser desorption/ionization (MALDI) FTMS experiments are performed on an IonSpec FTMS mass spectrometer. Electrospray ionization (ESI) mass spectrometry experiments were performed on an API 100 Perkin Elmer SCIEX single quadrupole mass spectrometer.

\section{Spectral Data for MPEG Tartrate Ligands:}

MPEG 350 Tartrate Ligand (1d): ${ }^{1} \mathrm{H}$ NMR $\left(\mathrm{CDCl}_{3}\right): \delta 4.69(\mathrm{~s}, 2 \mathrm{H}), 4.49-4.40(\mathrm{~m}$, $4 \mathrm{H}), 3.76-3.79(\mathrm{~m}, 8 \mathrm{H}), 3.72-3.63(\mathrm{~m}, 90 \mathrm{H}), 3.59-3.57(\mathrm{~m}, 8 \mathrm{H}), 3.42(\mathrm{~s}, 12 \mathrm{H}) .{ }^{13} \mathrm{C}$ NMR $\left(\mathrm{CDCl}_{3}\right): \delta 171.9,72.9,72.7,72.3,71.0,70.8,69.2,65.3,59.5,53.9$. 
Monomethyltetraethyleneglycol Tartrate Ligand (1e): ${ }^{1} \mathrm{H} \mathrm{NMR}\left(\mathrm{CDCl}_{3}\right): \delta 4.58(\mathrm{~s}$, 2H), 4.39-4.29 (m, 4H), 3.69-3.66 (m, 8H), 3.61-3.55 (m, 12H), 3.51-3.48 (m, 8H), 3.32 $(\mathrm{s}, 8 \mathrm{H}) .{ }^{13} \mathrm{C} \mathrm{NMR}\left(\mathrm{CDCl}_{3}\right): \delta 171.4,72.5,72.4,71.8,70.5,68.7,64.8,61.6,59.0,53.5$. ESI-TOF for $\mathrm{C}_{22} \mathrm{H}_{42} \mathrm{O}_{14}\left(\mathrm{M}+\mathrm{H}^{+}\right)$calcd. 531.2653; found 531.2640.

Monomethyltriethyleneglycol Tartrate Ligand (1f): ${ }^{1} \mathrm{H} \mathrm{NMR}\left(\mathrm{CDCl}_{3}\right): \delta 4.70(\mathrm{~s}$, $2 \mathrm{H}), 4.51-4.42(\mathrm{~m}, 4 \mathrm{H}), 3.81-3.77(\mathrm{~m}, 8 \mathrm{H}), 3.76-3.66(\mathrm{~m}, 30 \mathrm{H}), 3.63-3.60(\mathrm{~m}, 8 \mathrm{H}), 3.44$ (s, 12H). ${ }^{1} \mathrm{H}$ NMR $\left(\mathrm{CDCl}_{3}\right): \delta 171.9,72.9,72.8,72.3,71.0,70.9,70.7,69.2,65.3,62.1$, 59.4. ESI-TOF for $\mathrm{C}_{18} \mathrm{H}_{34} \mathrm{O}_{12}\left(\mathrm{M}+\mathrm{Na}^{+}\right)$calcd. 465.1942; found 465.1948 .

Monomethyldiethyleneglycol Tartrate Ligand (1g): ${ }^{1} \mathrm{H}$ NMR $\left(\mathrm{CDCl}_{3}\right): \delta 4.71(\mathrm{~s}$, $2 \mathrm{H}), 4.55-4.43(\mathrm{~m}, 4 \mathrm{H}), 3.82-3.80(\mathrm{~m}, 4 \mathrm{H}), 3.72-3.70(\mathrm{~m}, 4 \mathrm{H}), 3.63-3.61(\mathrm{~m}, 4 \mathrm{H}), 3.45$ (s, 6H). ${ }^{13} \mathrm{C} \mathrm{NMR}\left(\mathrm{CDCl}_{3}\right): \delta 171.9,72.6,72.3,70.8,69.2,65.3,59.5$. ESI-TOF for $\mathrm{C}_{14} \mathrm{H}_{26} \mathrm{O}_{10}\left(\mathrm{M}+\mathrm{Na}^{+}\right)$calcd. 377.1418; found 377.1416.

Monomethylethyleneglycol Tartrate Ligand (1h): ${ }^{1} \mathrm{H} \mathrm{NMR}\left(\mathrm{CDCl}_{3}\right): \delta 4.72(\mathrm{~s}, 2 \mathrm{H})$, 4.52-4.43 (m, 4H), 3.72-3.69 (m, 4H), $3.46(\mathrm{~s}, 6 \mathrm{H}) .{ }^{13} \mathrm{C} \mathrm{NMR}\left(\mathrm{CDCl}_{3}\right): \quad \delta$ 171.9, 72.5, 70.5, 65.5, 59.4. ESI-TOF for $\mathrm{C}_{10} \mathrm{H}_{18} \mathrm{O}_{8}\left(\mathrm{M}+\mathrm{H}^{+}\right)$calcd. 267.1080; found 267.1062.

ROESY Experiments: Ligand-Ti complexes were prepared in $\mathrm{CD}_{2} \mathrm{Cl}_{2}$ in an identical manner as used in asymmetric epoxidation reactions. Upon preparation, tubes were flame sealed and warmed to room temperature for 10 minutes. ROESY spectra were acquired at $-20{ }^{\circ} \mathrm{C}$ using 512 increments and 48 scans per increment. The mixing time was set at $200 \mathrm{~ms}$. 
Molecular Weight Determinations: Molecular weight determinations were performed by the Signer method ${ }^{1}$ using tetra- $n$-butyltin as the molecular weight standard. In a typical experiment, tartrate and $\mathrm{Ti}(\mathrm{OiPr})_{4}$ were combined in $\mathrm{CH}_{2} \mathrm{Cl}_{2}(3 \mathrm{~mL})$ on one side of the apparatus, while $\mathrm{SnBu}_{4}$ in $\mathrm{CH}_{2} \mathrm{Cl}_{2}(3 \mathrm{~mL})$ was added to the opposite side. After addition, the solvent was degassed via several freeze-pump-thaw cycles, and the apparatus allowed to equilibrate under vacuum in a draft-free area. Experiments typically took 7 days to reach equilibrium.

${ }^{1}$ (a) Clark, E. P. Ind. Eng. Chem., Anal. Ed. 1941, 13, 820-821. (b) Burger, B. J.; Bercaw, J. E. in Experimental Organometallic Chemistry: A Practice in Synthesis and Characterization. ACS Symposium Series 357 (Eds.: A. L. Wayada, M. Y. Darensbourg), American Chemical Society, Washington, DC, 1987, pp. 94-96. 


\section{1c}

Nu

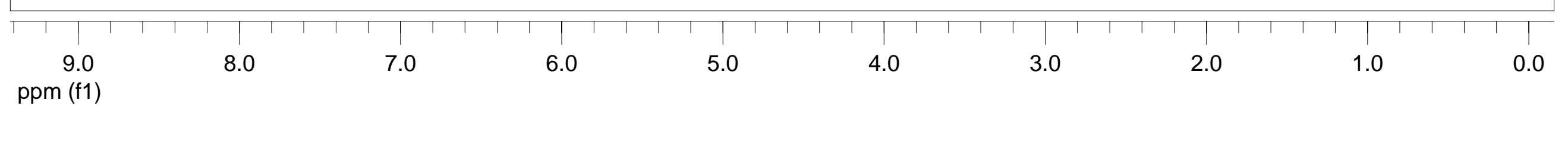


1c

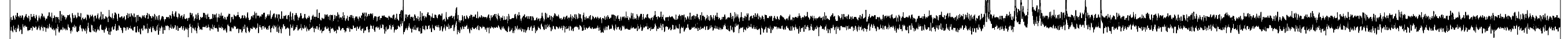

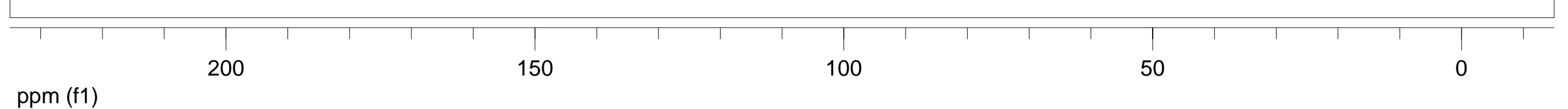

ppm (f1) 


\section{MALDI-TOF REFLECTRON}

Original Filename: e:Idatalroutinel2004Inov04|110404|3016aw.ms

This File \# 2 : E:IDATAIROUTINEI2004INOV04I110404ISMOOTH.MS

Comment:

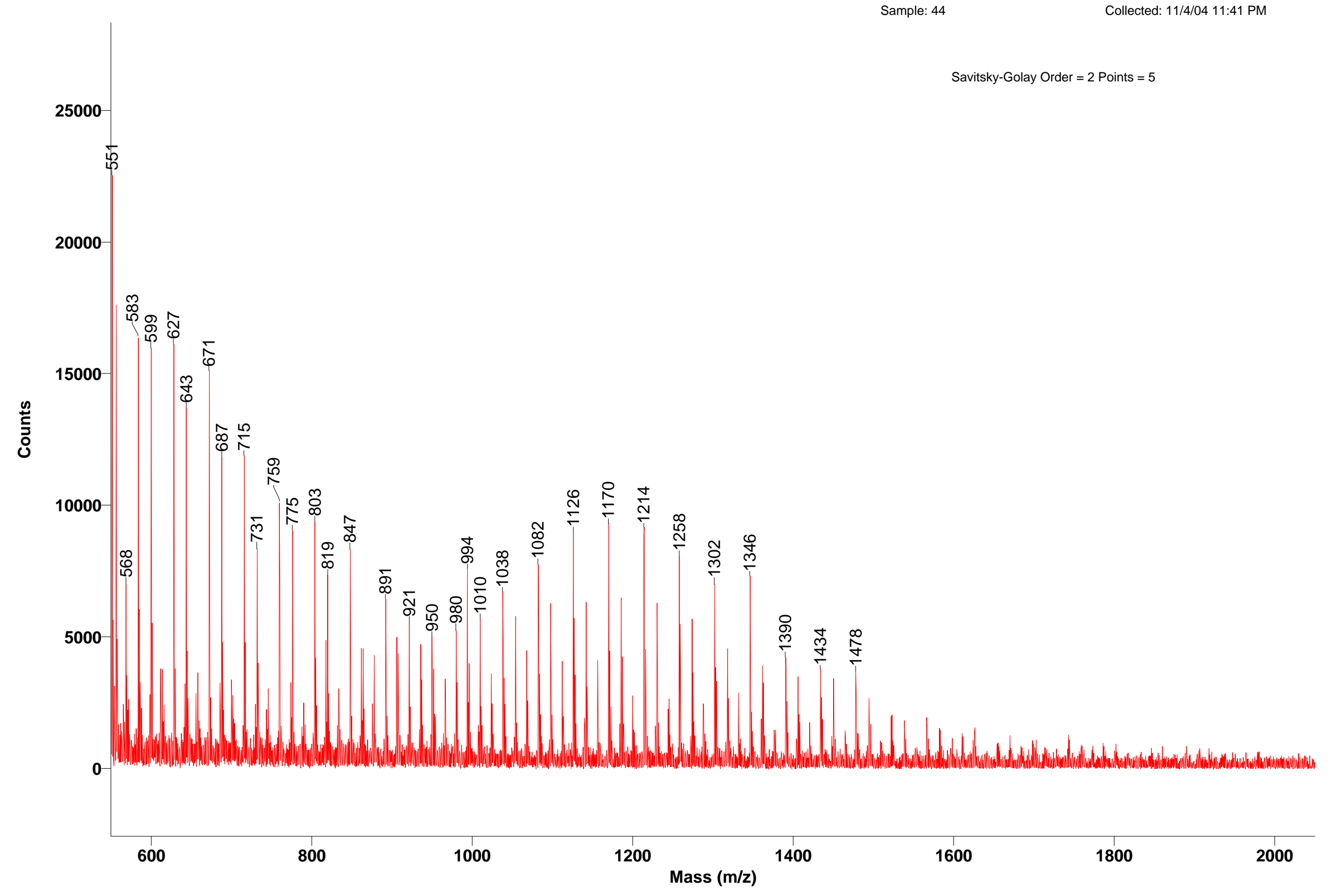

Method: R2000KH

Mode: Reflector

Accelerating Voltage: 20000

Grid Voltage: $72.000 \%$

Guide Wire Voltage: $0.030 \%$

Delay: 75 ON

Laser : 2140

Pressure: $2.41 \mathrm{e}-07$

Low Mass Gate: 400.0

Timed Ion Selector: 379.0 OFF

Negative lons: OFF

Collected: 11/4/04 11:41 PM
Scans Averaged: 110 
1d

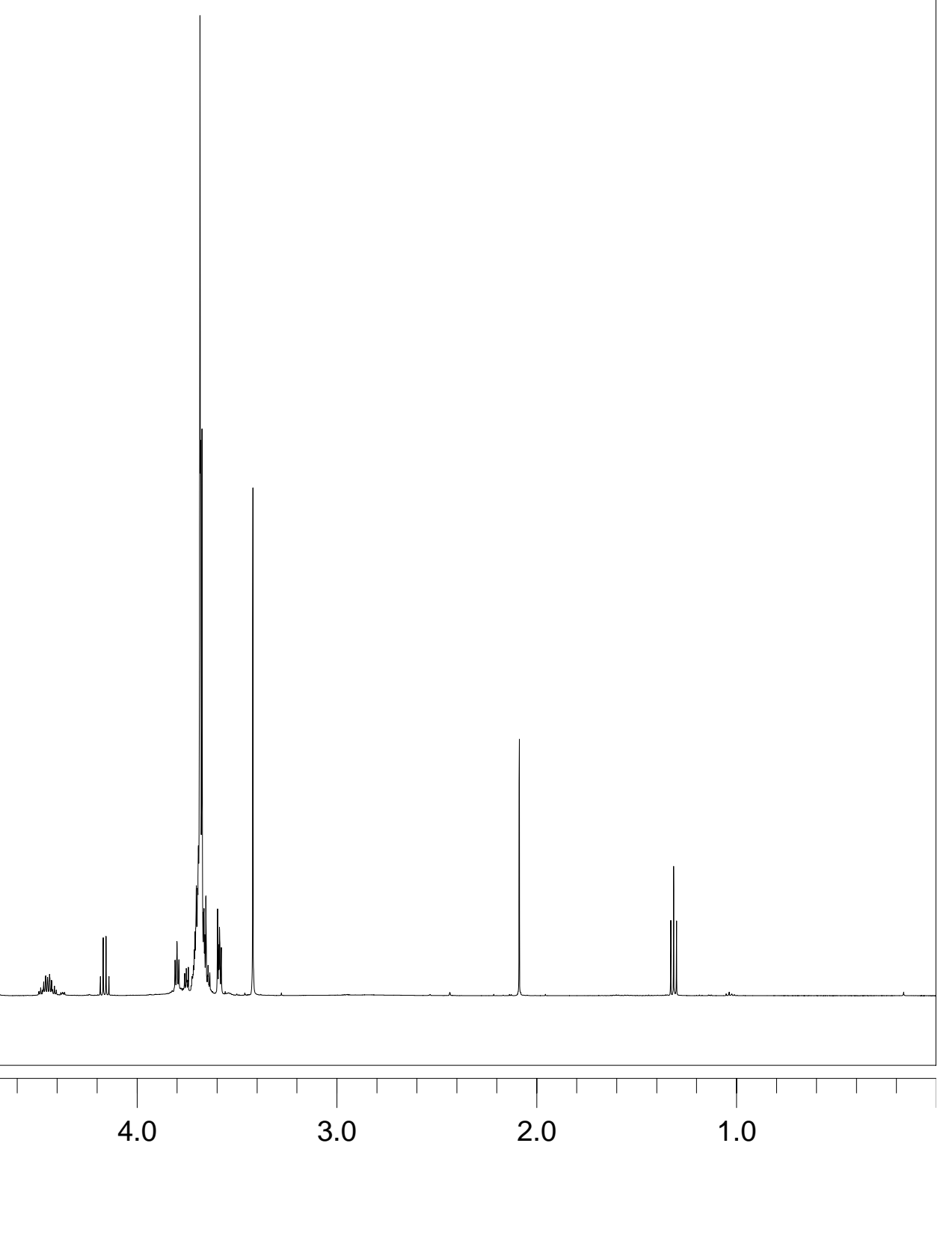




\section{MALDI-TOF REFLECTRON}

Original Filename: e:Idatalroutinel2004Inov041110404|3019aw.ms

This File \# 2 : E:IDATAIROUTINEI2004INOV041110404ISMOOTH.MS

Comment:

40000

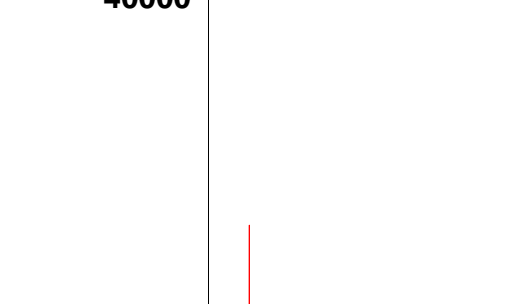

\section{$1 d$}

Method: R2000KH

Mode: Reflector

Accelerating Voltage: 20000

Grid Voltage: $72.000 \%$

Guide Wire Voltage: $0.030 \%$

Delay: 75 ON

Sample: 45
Laser : 2000

Scans Averaged: 81

Pressure: $2.30 \mathrm{e}-07$

Low Mass Gate: 400.0

Timed Ion Selector: 379.0 OFF

Negative lons: OFF

Collected: 11/4/04 11:46 PM

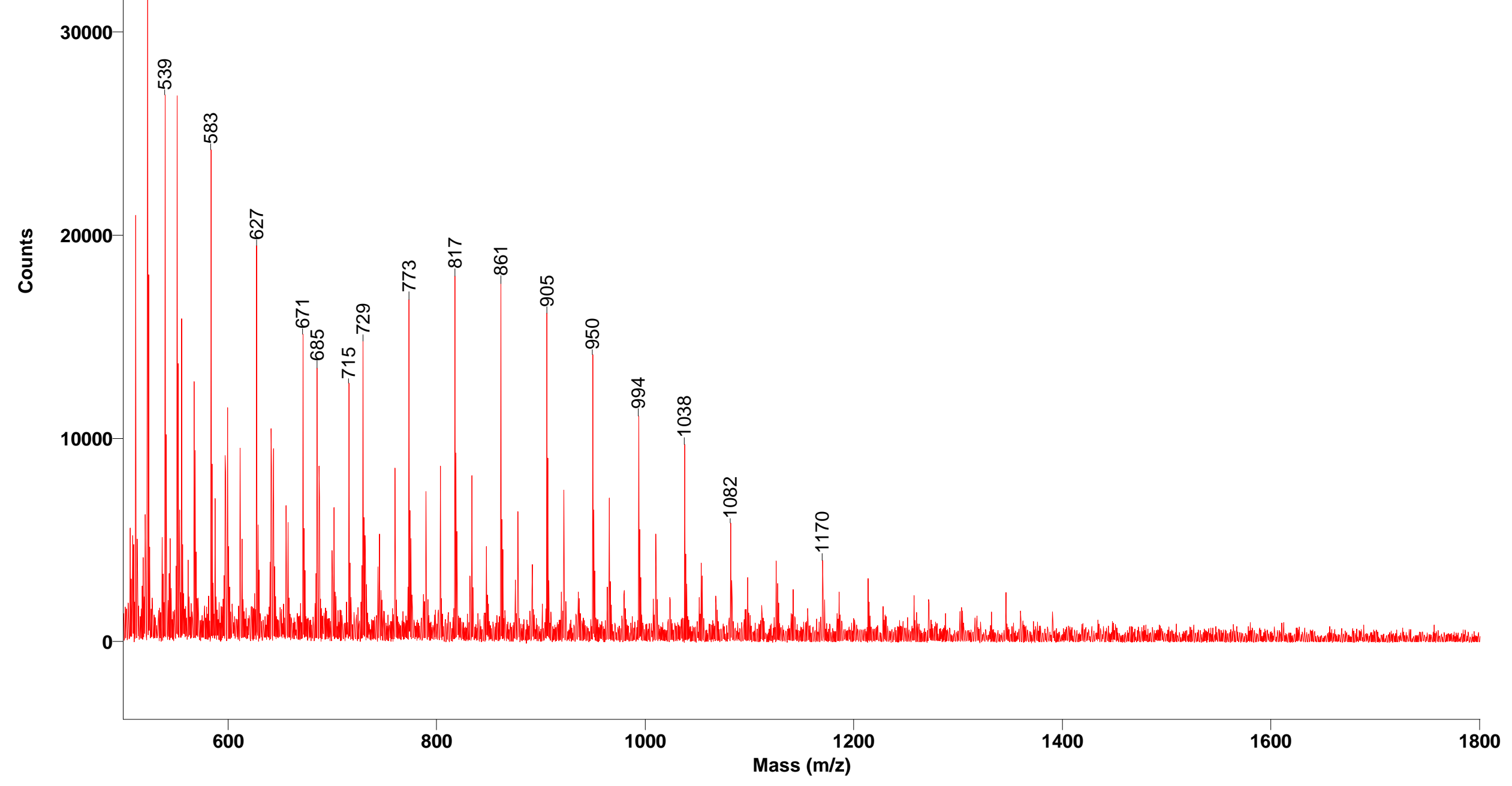


$1 e$

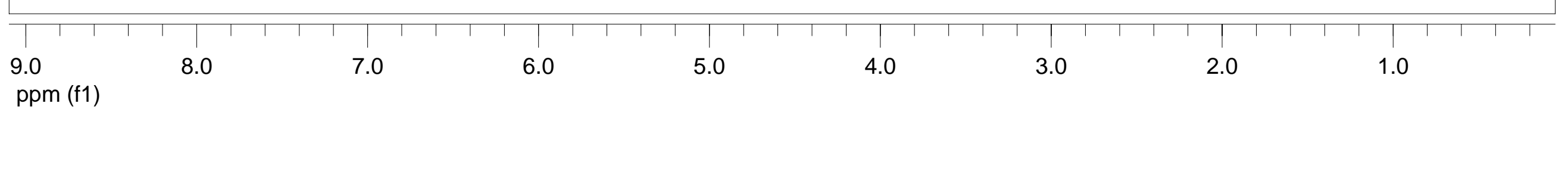




\section{$1 f$}

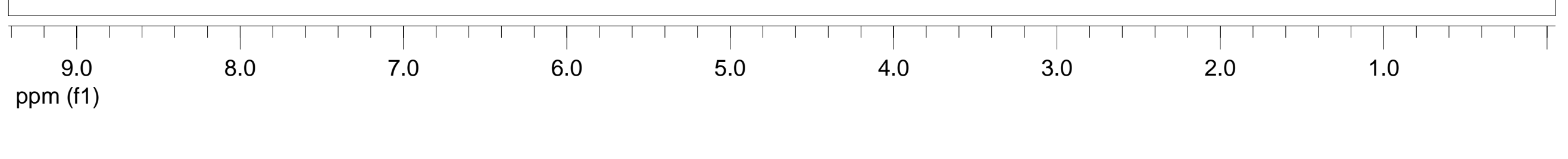




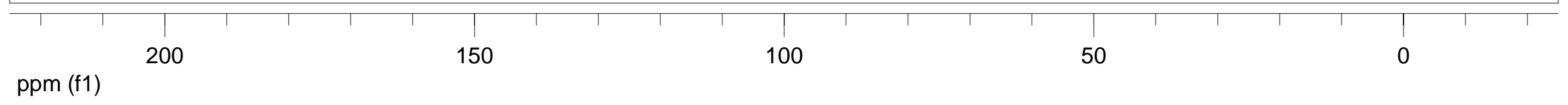

ppm (f1) 
$1 g$

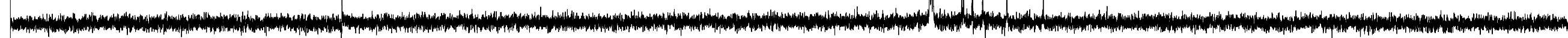

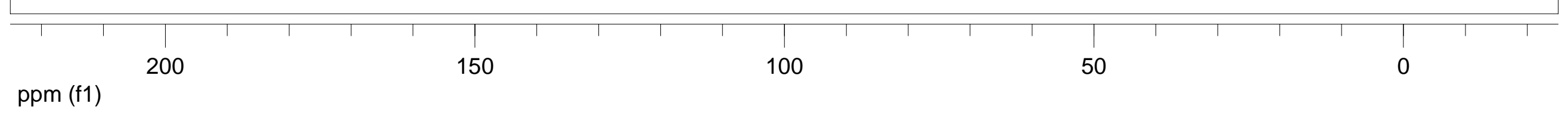


1h

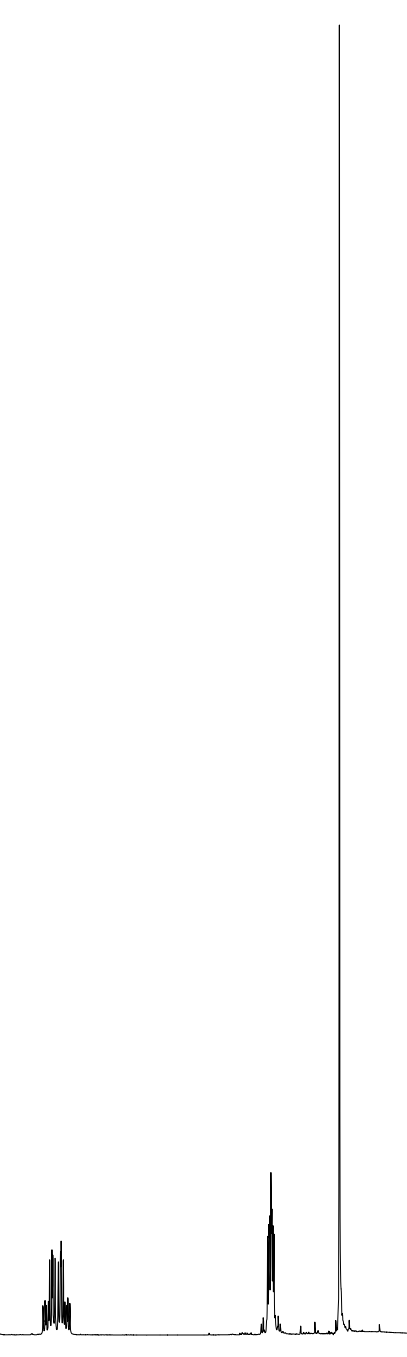

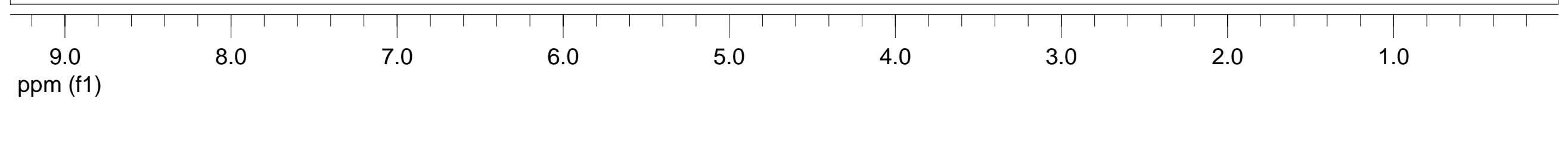




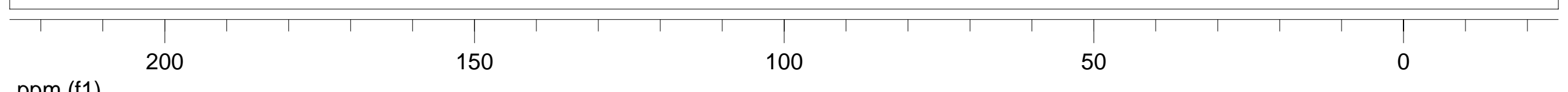
ppm (f1) 